\title{
YOU'VE BEEN FRAMED! STUDYING PEOPLE CHOOSING OPTIONS
}

\author{
Mairéad Hogan and Chris Barry \\ NUI Galway \\ Galway, Ireland
}

\begin{abstract}
This paper presents a research project that used eye tracking technology to study optionality within the online transactional process. The focus was on how micro-decisions can be framed: the option type; the impact of decision default values; and the types of decision constructs. It elaborates and improves on a pilot study that was used to test the experiment design. Prior research that identified problematic decision constructs informed the types of decisions studied. The main findings relate to participant task error rates.
\end{abstract}

\section{KEYWORDS}

Micro-Decision, Opt-in, Opt-out, Framing, Default Effect, Eye Tracking

\section{INTRODUCTION}

Opt-in rates on commercial websites are generally low, to the frustration of firms and marketeers. An opt-in is generally accepted as requiring express permission by an individual to allow the provision of some service or product, while an opt-out is where a course of action is pre-selected and the user must expressly deny that permission. The Cambridge Dictionary (Cambridge Dictionary, 2019) describes opt-in as "the fact of choosing to take part in an activity, arrangement, etc. rather than being forced to take part". The same source describes an opt-out as "to choose not to be part of an activity or to stop being involved in it." Quick 'micro-decisions' are usually presented to users in the form of an opt-in or opt-out. Frustrated consumers have become accustomed to dealing with numerous opt-in and opt-out services and charges as they negotiate transactional processes. In a paper describing certain design practices in the low cost carrier (LCC) sector significant disquiet was expressed amongst consumers in respect of the number and ambiguous nature of a series opt-in and opt-out services, many unavoidable, once they had committed to purchasing a flight (Barry et al., 2011).

This paper describes a study of optionality focusing on how designers can frame decisions, the impact of default values, and the types of decision constructs. The analysis details the impact of each of these on errors in participant choice. Other aspects of the findings, such as cognitive load and gender effects, will be reported upon in later work. The findings reveal some exciting insights into the effect the presentation of choice by firms can have on consumer outcomes.

\section{CHOICE AND OPTIONALITY}

\subsection{Choice Framing}

The theory of rational choice, that has had such a tight hold over much research governing social and economic behaviour, has been questioned across a number of disciplines. Simon (1957) was among the first to signal its limitations by proposing the concept of 'bounded rationality'. Subsequently, Tversky and Kahneman (1981) theorised framed information could be encoded positively or negatively. Their research indicated the manner in which choice is framed to individuals significantly influences decision-making. They concluded the dependence of preferences on the formulation of decision problems constitutes a major concern for the theory 
of rational choice. Samuelson and Zeckhauser (1988) found decisions are often presented with 'influential labels', whereby there is nearly always one alternative that carries the label 'status quo'. In a series of experiments designed to test for status quo effects, they concluded decision-makers exhibited a significant choice bias towards the status quo. This also known as the default effect.

The default effect has been well documented (Johnson and Eagly, 1989; Ahmetoglu et al., 2010; Ploug et al., 2012; Anaraky et al., 2018). This is a phenomenon whereby the user is more likely to choose the default option when presented with a choice. In the case of opt-outs, this can result in users making an inadvertent purchase or accidently signing up to a mailing list. In the case of an opt-in, the default option is to decline to purchase or to sign up to a mailing list. While there is research recognising opt-out decisions can be problematic, with users often inadvertently making a purchase, or opting in to a mailing list (Bellman et al., 2001), there is little research on negative consequences of errors with opt-ins in the transactional process. In fact, opt-ins are generally seen as the more benign option, with the EU requiring the use of opt-ins for all distance purchasing European Union (2011).

\subsection{Dimensions of Option Presentation}

The presentation of options to consumers in contemporary B2C interactions is made up of a number of dimensions. Much of the research discussed earlier on framing largely related to a singular dimension, that of a polar decision, choosing one of two options. In the more sophisticated world of online consumerism, the presentation of choice and optionality can be greatly finessed. The presentation of an option may now have multiple, even layered, dimensions. Previous research (Hogan et al., 2014; Torres et al., 2014) determined options tend to be presented to the user in a variety of ways. Some options are straightforward with easy to understand defaults and choices, while other options are more complicated and require effort to decipher so as to identify the default and the action required to achieve the desired outcome. In addition, some options are simply presented to the consumer while others incorporate various levels of persuasion, presumably to encourage selection of the vendor's preferred outcome.

A desk analysis of 57 websites was conducted to determine the fundamental dimensions of option presentation for opt-out decision constructs in use in B2C websites. A total of 42 opt-outs were encountered across 17 of the websites examined. A number of dimensions were identified as contributing to option presentation: control type (e.g., checkbox, radio button or drop-down menu); default value (i.e., un-selected or pre-selected); question or information framing (i.e., acceptance, rejection or neutral language); general purpose of the construct (e.g., immediate revenue generation or permission to retain data); and additional persuaders (e.g., benefits of choosing the option or reassurance of privacy). In this study the default value and framing are the key variables examined. The work is restricted to checkboxes as these are the most commonly encountered control types for micro-decisions within the commercial transactional process.

\subsection{Option Framing}

Bellman et al. (2001) explored the impact of question framing on user decisions. In querying how consumers have unknowingly opted-in to something, they explored the tactics some firms employ to encourage consent. They identified different ways in which consent can be obtained and concluded there are consequential effects in how questions are presented to consumers. Indeed, by using the correct combination of question framing and default answers, firms 'can almost guarantee' consent. Lai and Hui (2006) also conducted research into the impact of question framing on user decisions. Their study indicates the manner in which the option is described, as well as the default option (i.e., checked or unchecked), has an impact on user choice. They found for opt-in decisions using checkboxes, users are more likely to accept an un-selected opt-in over a pre-selected opt-in. They suggest the positive language of acceptance is likely to influence the users' decision.

Question framing has also been shown to affect user choice (Tversky and Kahneman, 1981; Levin et al., 1998; Kuo et al., 2009; Anaraky et al., 2018), with positively framed messages more likely to result in the user opting in to the choice. However, most of the studies presented the choice to the user in a way that either emphasised the positive or negative consequences of accepting or rejecting the choice. Lai and Hui (2004) did examine the impact of acceptance and rejection framing as defined in this paper, along with the impact of the default value (either pre-selected or un-selected). They determined opt-ins were more likely to result in a positive selection when acceptance framing was coupled with an un-selected presentation. They identified no significant difference for opt-outs. 


\subsection{Using Opt-out Structures for Altruistic and Self-serving Outcomes}

There have been cogent arguments for the use of opt-out structures to persuade or nudge people into making positive choices such as the consumption of better foods like whole wheat (Van Kleef et al., 2018), renewable energy (Momsen and Stoerk, 2014), organ donation (Johnson and Goldstein, 2003; Van Dalen and Henkens, 2014), climate change (Ebeling and Lotz, 2015), green environment (Schubert 2017), environmental conservation in hotels (Ebeling and Lotz, 2015), health surveys (Johnson et al., 2002) and retirement savings (Madrian and Shea, 2001; Beshears et al., 2009).

It has been observed that in recent years that some firms in particular sectors such as airlines, online car hire firms and comparison sites such as hotel reservations have been using design techniques that are not just unorthodox but of apparent deliberate design with a variety of self-serving ambitions. These include: making options unclear or ambiguous, leading consumers to make unnecessary choice; or deconstructing a final price with taxes, charges and items presented as options but which are, in fact, essential elements of the product or service (Barry et al., 2011; Barry et al., 2014).

\section{RESEARCH APPROACH}

As noted in Barry et al. (2016), eye tracking technology has been used extensively in web usability studies, alongside think-aloud techniques. This research project was conducted in two parts. In the first set of experiments on opt-out decision constructs, data was collected from 114 participants, 456 experiment trials and 23 Cued Retrospective Think-Aloud (RTA) sessions. The second set concerned opt-in decision constructs and collected data from 51 participants, 204 experiment trials and 12 Cued RTA sessions. The project was designed like this to control for construct type bias and potential confusion. This design was chosen from experiences with an earlier pilot study (Hogan et al., 2015). Thus statistical analysis was conducted, where relevant, on a between-subjects basis. The study was made up of three dimensions: an eye tracking experiment; self-assessment evaluations (subjective cognitive load); and Cued RTA sessions.

\subsection{Eye Tracking Experiment}

As the pilot study had highlighted the risks associated with the use of the eye tracking technology, significant effort was spent planning the experiments. Each of the variants of opt-out and opt-in decisions were examined: Un-selected Rejection Framing; Pre-selected Acceptance Framing; Pre-selected Rejection Framing; Pre-selected Neutral Framing; Un-Selected Acceptance Framing; and Un-Selected Neutral Framing (see Table 1).

Table 1. Decision constructs types

\begin{tabular}{lcccc}
\hline Construct Name & $\begin{array}{c}\text { Construct } \\
\text { Type }\end{array}$ & Default Value & Framing & Presented In \\
\hline Un-Selected Rejection & USR & Un-selected & Rejection & Opt-in and Opt-out \\
\hline Pre-Selected Acceptance & PSA & Pre-selected & Acceptance & Opt-in and Opt-out \\
\hline Pre-Selected Rejection & PSR & Pre-selected & Rejection & Opt-out \\
\hline Pre-Selected Neutral & PSN & Pre-selected & Neutral & Opt-out \\
\hline Un-Selected Acceptance & USA & Un-selected & Acceptance & Opt-in \\
\hline Un-Selected Neutral & USN & Un-selected & Neutral & Opt-in \\
\hline
\end{tabular}

The opt-out and opt-in decisions presented to participants were similar to real-world micro-decisions ordinarily facing users when engaged in a commercial transactional process on websites (Barry et al., 2014; Barry et al., 2016). 
In each study, participants were presented with either four opt-in or four opt-out micro-decisions. Participants were told to buy the product in two instances and not to buy in the other two. For the opt-in decisions, the participant needed to take action to make a purchase. In the case of the opt-in, the default option was to decline the purchase. For opt-out decisions, the participant needed to take action to decline the purchase, with the default option being to purchase the item. The order of presentation of the four screens was randomized when presented to participants. The core webpage screen was a breakdown insurance product to which participants were asked if they require an enhanced monthly-costed, add-on feature. Each screen had a decision point with a checkbox beside it (see Table 2).

Table 2. Decision constructs presented to participants

\begin{tabular}{ll}
\hline $\begin{array}{l}\text { Construct } \\
\text { Type }\end{array}$ & Decision Construct \\
\hline PSA - opt-out & $\square$ Rescue Plus includes free car hire and travel expenses. I want to purchase Rescue Plus. \\
\hline PSN - opt-out & $\square$ Rescue Plus. \\
\hline PSR - opt-out & $\square$ Rescue Plus includes free car hire and travel expenses. If you would rather not purchase Rescue Plus, please untick this box. \\
\hline USR - opt-out & $\square$ Rescue Plus includes free car hire and travel expenses. If you would rather not purchase Rescue Plus, please tick this box. \\
\hline USA - opt-in & $\square$ Rescue Plus. \\
\hline USN - opt-in & $\square$ Rescue Plus includes free car hire and travel expenses. If you would rather not purchase Rescue Plus, please leave this box unticked. \\
\hline USR - opt-in &
\end{tabular}

\section{EXPLORATORY ANALYSIS}

Once the data was gathered, exploratory analysis was conducted. The main focus of this analysis was participant error rates. A participant is deemed to have made an error when they make a mistake in the decision they have been told to take. For example, when told to 'Buy Rescue Plus Insurance' by choosing to tick or untick a checkbox, they unknowingly take the incorrect action. The error rates for opt-in and opt-out constructs were compared and contrasted. Framing and default value were also examined.

\subsection{Overall Error Rate}

The first step in exploring our data was to examine the error rate for the different construct types.

Table 3. Error rate for opt-out constructs

\begin{tabular}{cccc}
\hline Construct Type & Correct & Incorrect & Total \\
\hline PSA & $94(82 \%)$ & $20(18 \%)$ & 114 \\
\hline PSN & $87(76 \%)$ & $27(24 \%)$ & 114 \\
\hline PSR & $86(75 \%)$ & $28(25 \%)$ & 114 \\
\hline USR & $72(63 \%)$ & $42(37 \%)$ & 114 \\
\hline Total & $339(74 \%)$ & $117(26 \%)$ & 456 \\
\hline
\end{tabular}

For opt-outs (see Table 3) the number of incorrect selections varied between the construct types, with PSA having the highest rate of correct selections and USR having the highest rate of incorrect selections. Overall, the rate of incorrect selections made by participants was quite high, ranging from $18 \%$ (PSA) to $37 \%$ (USR). Chi-square test was conducted to determine whether the relationship between construct type and error rate was significant. The test indicated significance $\left(\chi^{2}(3, \mathrm{~N}=456)=11.715, \mathrm{p}<0.01\right)$. However, the value for $\phi$ was 0.158 , indicating the association was weak, only accounting for $2.5 \%$ of the variation. 
Table 4. Error rate for opt-in constructs

\begin{tabular}{cccc}
\hline Construct Type & Correct & Incorrect & Total \\
\hline USN & $43(84 \%)$ & $8(16 \%)$ & 51 \\
\hline USA & $41(80 \%)$ & $10(20 \%)$ & 51 \\
\hline USR & $36(71 \%)$ & $15(29 \%)$ & 51 \\
\hline PSA & $25(49 \%)$ & $26(51 \%)$ & 51 \\
\hline Total & $145(71 \%)$ & $59(29 \%)$ & 204 \\
\hline
\end{tabular}

For opt-ins (see Table 4) the number of incorrect selections also varied between the construct types, with PSA having the highest rate of incorrect selections and USN having the highest rate of correct selections. Overall, the rate of incorrect selections made by participants was quite high, ranging from $16 \%$ (USN) to $51 \%$ (PSA). Chi-square test was conducted to determine if the relationship between construct type and error rate was significant. The test indicated significance $\left(\chi^{2}(3, \mathrm{~N}=204)=18.576, \mathrm{p}<0.01\right)$. However, the value for $\phi$ was 0.302 , indicating the association was relatively weak, only accounting for $9.1 \%$ of the variation. The error rate for PSA was almost double that of the next highest, suggesting this construct was particularly problematic.

\subsection{Opt-in Versus Opt-out Error Rate}

As can be seen in Tables 3 and 4 above, the error rate was high for both opt-in and opt-out constructs, with $26 \%$ of decisions on opt-out constructs incorrect, and $29 \%$ of decisions on opt-in constructs incorrect. The variation in error rates is wider for opt-ins (16-51\%) than for opt-outs (18-37\%). The two opt-in constructs with the highest error rates (PSA and USR) are rarely encountered in practice (Barry et al., 2014), with most opt-ins encountered being the more straightforward USA or USN. This is perhaps due to the lack of benefit associated with misinterpretation of an opt-in. With the opt-outs, the USR has a considerably higher error rate than the other constructs. Again, this construct type is less frequently encountered than the others ((Barry et al., 2014; Barry et al., 2016). However, it is still encountered relatively frequently, particularly in sign-ups for mailing lists. The higher prevalence of this opt-out construct may be due to the perceived advantages to firms associated with users selecting the option, whether intentionally or erroneously.

The error rate for opt-in and opt-out were compared using Chi-square. Opt-in had $71 \%$ correct and $29 \%$ incorrect while opt-out had $74 \%$ correct and $26 \%$ incorrect. No significant difference was seen between the opt-in and opt-out constructs. As the USR for opt-out and the PSA for opt-in are rarely encountered, the error rates were compared for the more commonly encountered constructs. Again, there was no significant difference detected. Previous research (Bellman et al., 2001; Lai and Hui, 2006; Ahmetoglu et al., 2010) has reported increased likelihood of users signing up or purchasing when presented with an opt-out, rather than an opt-in, finding framing and default value to impact on user decisions. The findings in this study, do not support that premise (a similar result was found by Abbink and Hennig-Schmidt (2006)), perhaps because participants had pre-decided their desired outcome rather than being undecided.

\subsection{The Effect of Default Action on Error Rate}

The data was analyzed to determine whether the default action impacted on participants accuracy when making micro-decisions. For the opt-out constructs, the default action was to buy the add-on whereas for the opt-in constructs, the default action was to not buy the add-on. Chi-square was used to determine whether there was a significant difference in the error rate, depending on whether the participant's instruction was to choose the default action for the construct or to take action to change the default action. The analysis indicated no significant difference in error rates $\left(\chi^{2}(1, \mathrm{~N}=204)=0.024, \mathrm{p}=0.877\right.$ for opt-in and $\chi^{2}(1, \mathrm{~N}=456)=2.587$, $\mathrm{p}=0.108$ for opt-out). This contradicts previous research indicating users tend to choose the default action when presented with a choice. However, in previous research (Johnson and Eagly, 1989; Ahmetoglu et al., 2010; Ploug et al., 2012; Anaraky et al., 2018) participants were allowed a free choice when presented with the options. The studies either examined rates of acceptance of the default action or presented the participants with a free choice. In this study, participants were told to buy or not buy so they had already made their decision 
regarding which action they would take. This suggests the default action only affects choice when the user has not already decided which action they wish to take. The default effect can be seen as an influencer for the undecided user, rather than a persuader for a change of mind.

\subsection{The Effect of Construct Design on Error Rate}

In this study the options were presented to participants using 3 different types of framing, as identified by the authors in previous research (Hogan et al., 2014). The frames (acceptance, rejection and neutral framing) are all commonly used in the presentation of micro-decisions on websites. Additionally, this study examines error rate when the user has pre-decided their course of action, rather than being undecided in their choice.

Lai and Hui (2004) suggest that the tick in a pre-selected checkbox can have an anchoring effect on users which results in amplification of the framing. Thus, a pre-selected checkbox with rejection framing acts as a negative anchor and is more likely to result in the user rejecting the option while a pre-selected checkbox with acceptance framing acts as a positive anchor and is more likely to result in the user accepting the option. If this were the case, one might see more errors for participants instructed to not buy than those instructed to buy for the PSA constructs and to see more errors for those instructed to buy than those instructed to not buy for PSR constructs. No significant difference was seen for either PSA or PSR when Chi-square tests were run. This suggests that while an anchoring effect may nudge a user towards a particular decision, it will not cause them to mistakenly choose an option if they have already decided.

\subsection{The Effect of Framing on Error Rate}

As default actions can impact on the users' selection, the framing of opt-in and opt-out constructs was examined separately as they have different default actions. As can be seen in Table 5, acceptance framing had the highest rate of correct selections, followed by neutral framing, with rejection framing having the highest rate of incorrect selections. A Chi-square test indicated that the framing had a significant effect on the error rate $\left(\chi^{2}(2, \mathrm{~N}=456)=7.209, \mathrm{p}=0.027\right)$. However, the value for $\phi$ was 0.126 , indicating the association was weak, only accounting for $1.6 \%$ of the variation. Although the anchor effect did not have a significant effect on the error rate, the one un-selected construct type was eliminated from the test as it may have impacted on the result when coupled with framing. No significant difference was seen, suggesting that framing on its own does not impact on error rate for opt-out constructs.

Table 5. Error rate for opt-out constructs

\begin{tabular}{cccc}
\hline Framing & Correct & Incorrect & Total \\
\hline Acceptance & $94(82 \%)$ & $20(18 \%)$ & 114 \\
\hline Neutral & $87(76 \%)$ & $27(24 \%)$ & 114 \\
\hline Rejection & $158(69 \%)$ & $70(31 \%)$ & 228 \\
\hline Total & $339(74 \%)$ & $117(26 \%)$ & 456 \\
\hline
\end{tabular}

The tests were repeated for opt-ins (see Table 6). When all constructs were considered, a significant difference was seen $\left(\chi^{2}(2, \mathrm{~N}=204)=6.637, \mathrm{p}=0.041\right)$. However, the value for $\phi$ was 0.177 , a weak association, only accounting for $3.1 \%$ of the variation. The test was repeated using only the un-selected opt-ins. No significant difference was seen, suggesting framing on its own does not impact error rate for opt-in constructs.

Table 6. Error rate for opt-in constructs

\begin{tabular}{cccc}
\hline Framing & Correct & Incorrect & Total \\
\hline Acceptance & $66(65 \%)$ & $36(35 \%)$ & 102 \\
\hline Neutral & $43(84 \%)$ & $8(16 \%)$ & 51 \\
\hline Rejection & $36(71 \%)$ & $15(29 \%)$ & 51 \\
\hline Total & $145(71 \%)$ & $59(29 \%)$ & 204 \\
\hline
\end{tabular}




\section{CONCLUSIONS}

This study set out to examine whether the design of the constructs used for micro-decisions in online, commercial transactions impacted on error rate when the user had already decided on the desired outcome. The study participants were told to either buy or not buy the product. The error rate was high for all construct types, although some were considerably higher than others. The error rates tended to be higher for less frequently encountered construct types than frequently encountered ones. The PSA for opt-in had an error rate $(51 \%)$ of over three times the lowest error rate for opt-in (16\% for USN), suggesting that this construct type was in some way misleading the participant. It is unusual to encounter a pre-selected opt-in on a website so the participants may have assumed a pre-selected checkbox was an opt-out. The difference in error rates for the opt-outs was not as extreme, perhaps because of the higher prevalence of all types of opt-outs on commercial websites. Despite the difference in error rates between opt-in and opt-out constructs, no significant difference was found in the error rates, suggesting users are no more likely to erroneously select an option on opt-out than on opt-in.

The default effect is a phenomenon whereby users, when presented with options, are more likely to accept the default option than change it. In previous literature, study participants were allowed a free choice. In this study, participants were told what decision to make when presented with the construct. If the default effect impacted the outcome, a higher error rate would be expected when the participant was required to change from the default action. No significant difference was seen between the error rates, suggesting the default effect does not apply when the user has already decided on the course of action they wish to take. Therefore, while it may influence the undecided user, it is unlikely to dissuade the decided user from their planned course of action.

The anchoring effect of a pre-selected checkbox has been shown previously to reinforce the framing of a construct, whereby acceptance framing is more likely to result in option acceptance and rejection framing is more likely to result in option rejection. It was posited that this results from more time spent contemplating the text presented to the user. In this study, no anchoring effect was apparent, likely because participants had pre-decided on the course of action and so, were less susceptible to the nudge power of checkbox pre-selection.

Once the default value of the decision construct was controlled, the framing of the construct did not have a significant effect on the error rate for either opt-in or opt-out. This contradicts previous research which states that acceptance framing is more likely to result in the user accepting the option offered while rejection framing is more likely to result in the user rejecting the option offered. Again, this is likely to be related to the fact the participants had pre-decided on the desired course of action.

This study has added to the body of knowledge on the use of opt-in and opt-out constructs on commercial websites. Previous research suggests designing constructs in a particular way can increase the number of users accepting or rejecting the option presented. This study suggests design is unlikely to change the user's mind if they have already decided on a course of action. Therefore, if a designer wishes to persuade an already decided user along a particular path, methods of persuasion beyond reliance on construct design need to be considered.

\section{REFERENCES}

Abbink, K. and Hennig-Schmidt, H., 2006. Neutral versus loaded instructions in a bribery experiment. Experimental Economics, Vol. 9, No. 2, pp. 103-121.

Ahmetoglu, G. et al, 2010. Pricing Practices: Their Effects on Consumer Behaviour and Welfare. Mountainview Learning. University College London.

Anaraky, A. et al, 2018. Reducing Default and Framing Effects in Privacy Decision-Making. SIGHCI 2018 Proceedings.

Barry, C. et al, 2011. Low-Cost Carriers and High-Tech Barriers - User Views on Questionable Web Design Practices in Ireland. Irish Journal of Management, Vol. 31, No. 1, pp. 43-58.

Barry, C. et al, Year. Perceptions of Low Cost Carriers' Compliance with EU Legislation on Optional Extras. 20th International Conference on Information Systems Development, Edinburgh, Scotland.

Barry, C. et al, 2014. Confirming a Taxonomy of Decision Constructs in Business-to-Consumer Commercial Transactions. $23 r d$ International Conference on Information Systems Development, Varaždin, Croatia.

Barry, C. et al, 2014. Identifying essential and optional decision constructs in on-line transactional processes. Information System Development: Improving Enterprise Communication. M. J. Escalona, G. Aragón, H. Linger et al. New York, Springer.

Barry, C. et al, 2016. Framing or Gaming? Constructing a Study to Explore the Impact of Option Presentation on Consumers. Information System Development: Transforming Healthcare Through Information Systems, Springer International Publishing: 111-124. 
Bellman, S. et al, 2001. On site: to opt-in or opt-out?: it depends on the question. Communications of the ACM, Vol. 44, No. 2, pp. 25-27.

Beshears, J. et al, 2009. The importance of default options for retirement saving outcomes: Evidence from the United States. Social security policy in a changing environment, University of Chicago Press: 167-195.

Cambridge Dictionary, 2019. Opt-in.

Ebeling, F. and Lotz, S., 2015. Domestic uptake of green energy promoted by opt-out tariffs. Nature Climate Change, Vol. 5, No., pp. 868.

European Union, 2011. Directive on Consumer Rights

Hogan, M. et al, 2014. Theorising and testing a taxonomy of decision constructs. Journal of Customer Behaviour, Vol. 13, No. 3, pp. 171-185.

Hogan, M. et al, 2015. An Eye Tracking Pilot Study on Optionality - Some Lessons Learned. 17th Irish Academy of Management Annual Conference, Galway.

Johnson, B. T. and Eagly, A. H., 1989. Effects of involvement on persuasion: A meta-analysis. Psychological Bulletin, Vol. 106, No. 2, pp. 290-314.

Johnson, E. J. et al, 2002. Defaults, Framing and Privacy: Why Opting In-Opting Out1. Marketing Letters, Vol. 13, No. 1, pp. 5-15.

Johnson, E. J. and Goldstein, D. G., 2003. Do defaults save lives? Science, Vol. 302, No., pp. 1338-1339.

Kuo, F.-Y. et al, 2009. An exploratory study of cognitive effort involved in decision under Framing - an application of the eye-tracking technology. Decision Support Systems, Vol. 48, No. 1, pp. 81-91.

Lai, Y.-L. and Hui, K.-L., 2004. Luring the Prey: Attracting Online Consumer Participation through Choice Configurations. American Market Association (AMA) Marketing and Public Policy Conference, Washington D.C., USA.

Lai, Y.-L. and Hui, K.-L., Year. Internet opt-in and opt-out: investigating the roles of frames, defaults and privacy concerns. Proceedings of the 2006 ACM SIGMIS CPR conference on computer personnel research: Forty four years of computer personnel research: achievements, challenges \& the future, Claremount, California, USA, ACM.

Levin, I. P. et al, 1998. All frames are not created equal: A typology and critical analysis of framing effects. Organizational behavior and human decision processes, Vol. 76, No. 2, pp. 149-188.

Madrian, B. C. and Shea, D. F., 2001. The Power of Suggestion: Inertia in 401 (k) Participation and Savings Behavior*. The Quarterly journal of economics, Vol. 116, No. 4, pp. 1149-1187.

Momsen, K. and Stoerk, T., 2014. From intention to action: Can nudges help consumers to choose renewable energy? Energy Policy, Vol. 74, No., pp. 376-382.

Ploug, T. et al, 2012. To nudge or not to nudge: cancer screening programmes and the limits of libertarian paternalism. J Epidemiol Community Health, Vol. 66, No. 12, pp. 1193-1196.

Samuelson, W. and Zeckhauser, R., 1988. Status quo bias in decision making. Journal of risk and uncertainty, Vol. 1, No. 1, pp. 7-59.

Simon, H. A., 1957. A Behavioral Model of Rational Choice. Models of Man, Social and Rational: Mathematical Essays on Rational Human Behavior in a Social Setting. New York, Wiley.

Torres, A. M. et al, 2014. The Identification of Decision Constructs used in Online Transactional Processes. 27th BLED eConference.

Tversky, A. and Kahneman, D., 1981. The framing of decisions and the psychology of choice. Science, Vol. 211, No. 4481, pp. 453-458.

Van Dalen, H. P. and Henkens, K., 2014. Comparing the effects of defaults in organ donation systems. Social science \& medicine, Vol. 106, No., pp. 137-142.

Van Kleef, E. et al, 2018. The effect of a default-based nudge on the choice of whole wheat bread. Appetite, Vol. 121, No., pp. 179-185. 